\title{
Ground state and excitation dynamics in Ag doped helium clusters
}

\author{
Massimo Mella ${ }^{\text {a) }}$ \\ Dipartimento di Chimica Fisica ed Elettrochimica, Universita' degli Studi di Milano, via Golgi 19, \\ 20133 Milano, Italy \\ Maria Carola Colombo ${ }^{\text {b) }}$ and Gabriele Morosi ${ }^{\mathrm{c})}$ \\ Dipartimento di Scienze Chimiche, Fisiche e Matematiche, Universita' dell'Insubria, via Lucini 3, \\ 22100 Como, Italy
}

(Received 21 June 2002; accepted 11 September 2002)

\begin{abstract}
We present a quantum Monte Carlo study of the structure and energetics of silver doped helium clusters $\mathrm{AgHe}_{n}$ for $n$ up to 100. Our simulations show the first solvation shell of the Ag atom to include roughly $20 \mathrm{He}$ atoms, and to possess a structured angular distribution. Moreover, the ${ }^{2} P_{1 / 2} \leftarrow{ }^{2} S_{1 / 2}$ and ${ }^{2} P_{3 / 2} \leftarrow{ }^{2} S_{1 / 2}$ electronic transitions of the embedded silver impurity have been studied as a function of the number of helium atoms. The computed spectra show a redshift for $n \leqslant 15$ and an increasing blueshift for larger clusters, a feature attributed to the effect of the second solvation shell of He atoms. For the largest cluster, the computed excitation spectrum is found in excellent agreement with the ones recorded in superfluid He clusters and bulk. No signature of the direct formation of the proposed $\mathrm{AgHe}_{2}$ exciplex is present in the computed spectrum of $\mathrm{AgHe}_{100}$. To explain the absence of the fluorescent $D_{2}$ line in the experiments, a relaxation mechanism between the ${ }^{2} P_{3 / 2}$ and the ${ }^{2} P_{1 / 2}$ states is proposed on the basis of the partial overlap of the excitation bands in the simulated spectra. (C) 2002 American Institute of Physics. [DOI: 10.1063/1.1518472]
\end{abstract}

\section{INTRODUCTION}

Superfluid ${ }^{4} \mathrm{He}$ clusters represent a gentle environment where high resolution spectroscopic studies of atoms, atomic clusters, and molecules at low temperature can be carried out. ${ }^{1}$ In such cold and fluid quantum systems many perturbing effects due to the temperature and solid matrices are absent, making easier the interpretation of the experimentally recorded spectra. Moreover, their superfluid behavior allows interesting quantum effects to take place and to be experimentally probed (for instance see Refs. 2 and 3).

Whereas the coupling of the rotational and vibrational motion of the molecules with the quantum motion of the solvent is permitted by the similarity between energy levels, the electronic structure of an atom is characterized by energy differences orders of magnitude larger than the ones needed to induce excitation in the atomic motion. Although this difference might seem to work in the direction of simplifying the physical description of the electronic transition processes, many important details still wait to be clarified. As an example, the fluorescent $D_{2}$ emission line (i.e., the ${ }^{2} S_{1 / 2}$ $\leftarrow^{2} P_{3 / 2}$ radiative transition) of single valence electron heavy atoms dispersed in superfluid helium is absent, while the $D_{1}$ line $\left({ }^{2} S_{1 / 2} \leftarrow{ }^{2} P_{1 / 2}\right.$ transition) is sharp and only slightly shifted $(1-2 \mathrm{~nm})$ to the blue. ${ }^{4}$ This is in contrast with the large broadening and strong blueshift of the absorption lines. Moreover, some features of the LIF spectra of the dispersed

\footnotetext{
${ }^{a)}$ Electronic mail: Massimo.Mella@unimi.it

b) Present address: Laboratory of Inorganic Chemistry, ETH Hönggerberg, CH-8093 Zürich, Switzerland; Electronic mail: Colombo@inorg.chem.ethz.ch

${ }^{c}$ Electronic mail: Gabriele.Morosi@uninsubria.it
}

$\mathrm{Ag}$ were interpreted as signature of the $\mathrm{AgHe}$ and $\mathrm{AgHe}_{2}$ exciplexes formation. ${ }^{5}$

The blueshift and broadening of the absorption lines have been interpreted by means of a "bubble model." Here, the dispersed atom is enclosed in a spherical cavity due to the exchange repulsion of its valence electrons and the $\mathrm{He}$ ones. The liquid He around an atom is modeled by an isotropic sharp-edge density profile with no atomic internal structure. However, both the simple spherical bubble model ${ }^{6}$ and the one where quadrupolar distortions of the spherical cavity are allowed ${ }^{7}$ neither quantitatively predict the absorption spectrum of $\mathrm{Cs}$ and $\mathrm{Rb}$, nor allow one to interpret the small splitting of the $\operatorname{Rb} D_{2}$ line. Reasonably, the lack of any shell structure in the helium density profile, the absence of a full atomistic description during the excitation process, and the physically incomplete description of the bubble distortion by means of simple quadrupolar deformations may be held responsible for this undesirable outcome. ${ }^{8}$

In order to gain a better understanding of the excitation process and its dependency on the degree of "solvation" of the impurity, we feel a direct many-body simulation of the excitation spectra to be mandatory. This also allows one to explore the change in the spectra upon the increase of the number of He atoms in the clusters, and, at the same time, to test the validity of our theoretical approach.

With these goals in mind, we present a diffusion Monte Carlo study of the ${ }^{2} P_{3 / 2} \leftarrow{ }^{2} S_{1 / 2}$ and ${ }^{2} P_{1 / 2} \leftarrow{ }^{2} S_{1 / 2}$ absorption spectra of silver doped helium clusters. The $\mathrm{Ag}$ spectrum, both in bulk helium and in He clusters, has been deeply studied and well characterized ${ }^{5,9-11}$ showing that $\mathrm{Ag}$ is indeed solvated. Moreover, $a b$ initio interaction potentials between He and the excited ${ }^{2} P_{1 / 2},{ }^{2} P_{3 / 2}$, and ${ }^{2} S_{1 / 2}$ states of $\mathrm{Ag}$ are available. ${ }^{12}$ In principle, a thorough assessment of the 
quality of these potentials for the AgHe dimer would require computing a few relevant quantities and comparing the results with experimental data. To the best of our knowledge, no experiments directly probing the $\mathrm{AgHe}$ dimer properties have been published so far, and this hinders a direct comparison. However, on the basis of these potentials the broad band at $382 \mathrm{~nm}$ in the fluorescence spectrum was accurately reproduced and assigned to the $\mathrm{AgHe}$ and $\mathrm{AgHe}_{2}$ exciplexes, ${ }^{5}$ and the solvatation of the $\mathrm{Ag}$ atom inside the $\mathrm{He}$ clusters was correctly predicted. These two facts seem to indicate, although indirectly, the good accuracy of these $a b$ initio interaction potentials, so we chose to employ them in our many-body simulations.

\section{METHODS}

Pure and doped He clusters are characterized by their highly quantum nature, a feature that manifests itself in the small total binding energy and in the anharmonic motion of both the doping impurity and the $\mathrm{He}$ atoms. These facts rule out the possibility of using a semiclassical approach, that is to define an equilibrium geometry, found by minimizing the potential, whose energy is corrected for the zero point energy of the harmonic motion. On the other side, the size of the clusters does not allow for a quantum approach exploiting a basis set expansion as commonly done for few-body systems. To tackle the atomic description needed to compute the excitation spectra, we believe the quantum Monte Carlo (QMC) methods are the best suited techniques. Since these methods are well described in the literature, ${ }^{13}$ we restrain ourselves from presenting long discussions, except for the technical details that are relevant to the present work. In this respect, we simply state that the QMC family of methods could be partitioned into two branches with respect to the possibility of including or not including thermal effects in the simulation. The path integral Monte Carlo (PIMC) method allows one to sample the quantum thermal density matrix $\rho\left(\mathbf{R}^{\prime}, \mathbf{R}, \beta\right)$, so that finite temperature effects can be included, and to recognize more easily the onset of superfluid behavior. Conversely, the variational Monte Carlo (VMC) method allows one to optimize a trial wave function $\Psi_{T}(\mathbf{R})$ and to successively compute any expectation values $\langle O\rangle_{\mathrm{VMC}}$ from it, while the diffusion Monte Carlo (DMC) method corrects the remaining deficiencies of the variational description projecting out all the excited state components and samples $f(\mathbf{R})=\Psi_{0}(\mathbf{R}) \Psi_{T}(\mathbf{R})$, or less commonly $f(\mathbf{R})$ $=\Psi_{0}^{2}(\mathbf{R})$. Here, $\Psi_{0}(\mathbf{R})$ is the ground state wave function.

For the doped He clusters, the choice of a zero or a finite temperature method is dependent on the relative importance of the thermal excitation in the $\mathrm{He}$ atomic motion. Pure $\mathrm{He}$ clusters are usually very cold (their temperature is considered to be roughly $0.3-0.4 \mathrm{~K}$ ). For example, for $\mathrm{He}_{10}$, whose excitation gap between the ground and first vibrational excited state is estimated to be $1.7 \mathrm{~K}\left(1.2 \mathrm{~cm}^{-1}\right),{ }^{14}$ the population ratio is roughly 0.04 . For the $\mathrm{Ag}$ doped clusters, we anticipate that one should expect a larger gap with respect to the pure case on the basis of the AgHe interaction potential well, roughly $11 \mathrm{~cm}^{-1}(15 \mathrm{~K})$, and of its total energy of about $-4.0 \mathrm{~cm}^{-1}(-5.5 \mathrm{~K})$. If we made the conservative estimate

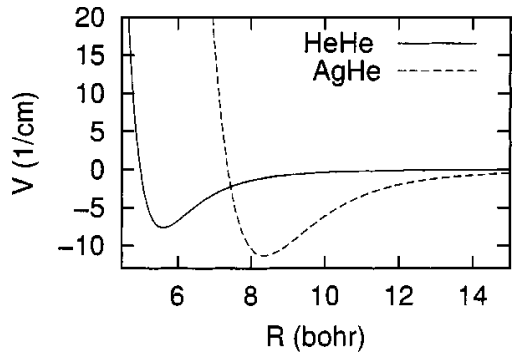

FIG. 1. $\mathrm{He}-\mathrm{He}$ and $\mathrm{Ag}-\mathrm{He}$ pair interaction potentials.

for the gap of $1.4 \mathrm{~cm}^{-1}(2 \mathrm{~K})$, the population ratio would drop down to 0.007 , therefore showing that thermal excitation, although present, should not modify substantially the results obtained by means of a zero temperature method. In conclusion, we select DMC as our method of choice. Nevertheless, we mention that PIMC should be the preferred method when one is interested in the local superfluid behavior around a dopant, or when structural properties could be modified by adding kinetic energy to the system due to heating. ${ }^{15}$

In atomic units, the Hamiltonian operator for our $\mathrm{AgHe}_{n}$ clusters reads as

$$
\mathcal{H}=-\frac{1}{2}\left(\sum_{i}^{n} \frac{\nabla_{i}^{2}}{m_{4} \mathrm{He}}+\frac{\nabla_{\mathrm{Ag}}^{2}}{m_{\mathrm{Ag}}}\right)+V(\mathbf{R}) .
$$

For the clusters with the silver atom in the ${ }^{2} S_{1 / 2}$ electronic ground state, we assume a potential of the form

$$
V(\mathbf{R})=\sum_{i<j}^{n} V_{\mathrm{HeHe}}\left(r_{i j}\right)+\sum_{i}^{n} V_{\mathrm{AgHe}}\left(r_{i}\right) .
$$

For $V_{\mathrm{HeHe}}\left(r_{i j}\right)$ we employed the Tang-Toennies-Yiu (TTY) potential, ${ }^{16}$ and for $V_{\mathrm{AgHe}}\left(r_{i}\right)$ we fitted the ${ }^{2} \Sigma$ AgHe potential by Jakubek and Takami ${ }^{12}$ by means of the analytical form

$$
V_{\mathrm{AgHe}}(r)=A \exp (-B r)+\frac{C}{r^{10}}+\frac{D}{r^{8}}+\frac{E}{r^{6}},
$$

where $A=3870264.2 \mathrm{~cm}^{-1}, \quad B=2.7637976 \AA^{-1}, \quad C$ $=12443020 \AA^{10} \mathrm{~cm}^{-1}, \quad D=2491338 \AA^{8} \mathrm{~cm}^{-1}, \quad$ and $E$ $=67213.355 \AA^{6} \mathrm{~cm}^{-1}$. Figure 1 shows both the TTY and the $V_{\mathrm{AgHe}}$ potentials in order to allow for a direct comparison. As a test of our fitted potential, we computed the energy of the $\mathrm{AgHe}$ dimer by means of a grid method ${ }^{17}$ obtaining -4.021 $\mathrm{cm}^{-1}$ : this value differs from the result of Ref. 12, -4.000 $\mathrm{cm}^{-1}$, by only $0.021 \mathrm{~cm}^{-1}$. No bound excited states were found.

Whereas, in principle, the two-body potential is only an approximation to the many-body one, this choice is justified on the basis of the perturbation theory. Since only a small charge transfer is present in $\mathrm{AgHe},{ }^{12}$ the main component of the interaction energy in the attractive region of the potential is the dispersion one. Due to the small dipolar and quadrupolar polarizabilities of $\mathrm{He}^{18}$ and to the short range dependency on the interatomic distances, the terms coming from three-body effects (e.g., induced dipole-induced dipoleinduced dipole and induced dipole-induced dipole-induced 
TABLE I. Total $\left(\mathrm{cm}^{-1}\right)$ and evaporation energy $\left(\mathrm{cm}^{-1} /\right.$ atom), $D_{1}$ and $D_{2}$ absorption wavelengths (nm) for $\mathrm{AgHe}_{n}$ clusters.

\begin{tabular}{rcccccc}
\hline \hline \multicolumn{1}{r}{$n$} & $E_{\mathrm{VMC}}(n)$ & $E_{\mathrm{DMC}}(n)$ & $\Delta(n)$ & $E_{\mathrm{VMC}} / E_{\mathrm{DMC}}$ & $D_{1}$ & $D_{2}$ \\
\hline Free Ag & & & & & 338.3 & 328.1 \\
1 & $-4.0243(2)$ & $-4.0212(9)$ & & 0.99 & & \\
2 & $-8.1724(3)$ & $-8.2333(5)$ & $4.212(1)$ & 0.99 & 344.5 & 334.8 \\
3 & $-12.450(1)$ & $-12.598(2)$ & $4.365(2)$ & 0.99 & 344.2 & 334.6 \\
4 & $-16.831(1)$ & $-17.112(1)$ & $4.514(2)$ & 0.98 & & \\
6 & $-25.966(2)$ & $-26.478(2)$ & $4.683(2)$ & 0.98 & 342.6 & 333.3 \\
8 & $-35.369(4)$ & $-36.259(4)$ & $4.891(2)$ & 0.97 & 341.3 & 332.3 \\
12 & $-54.213(7)$ & $-56.68(1)$ & $5.105(5)$ & 0.96 & 339.6 & 329.8 \\
13 & $-57.939(6)$ & $-61.78(1)$ & $5.10(1)$ & 0.94 & & \\
14 & $-62.184(8)$ & $-66.84(1)$ & $5.06(1)$ & 0.93 & & \\
15 & $-66.20(1)$ & $-71.61(5)$ & $4.77(5)$ & 0.92 & 338.3 & 328.5 \\
19 & $-78.60(4)$ & $-89.31(2)$ & $4.43(1)$ & 0.88 & & \\
20 & $-81.21(9)$ & $-93.17(3)$ & $3.86(4)$ & 0.87 & 337.3 & 327.6 \\
24 & $-94.28(8)$ & $-107.14(4)$ & $3.49(1)$ & 0.88 & & \\
25 & $-96.80(5)$ & $-110.3(1)$ & $3.2(1)$ & 0.88 & & \\
29 & $-110.62(5)$ & $-123.17(7)$ & $3.22(3)$ & 0.90 & & \\
30 & $-111.86(4)$ & $-126.11(7)$ & $2.9(1)$ & 0.89 & 336.2 & 326.6 \\
40 & $-140.22(8)$ & $-158.70(6)$ & $3.26(1)$ & 0.89 & 335.5 & 325.7 \\
50 & $-166.4(1)$ & $-191.3(3)$ & $3.26(3)$ & 0.87 & 334.7 & 324.9 \\
60 & $-197(1)$ & $-225.1(2)$ & $3.38(4)$ & 0.87 & 333.8 & 323.9 \\
70 & $-220(1)$ & $-259.9(4)$ & $3.48(4)$ & 0.85 & 333.4 & 323.6 \\
80 & $-245(1)$ & $-292.4(7)$ & $3.25(8)$ & 0.84 & 332.5 & 322.7 \\
90 & $-270(1)$ & $-326.2(7)$ & $3.4(1)$ & 0.83 & 331.9 & 322.0 \\
100 & $-289(2)$ & $-357.3(6)$ & $3.1(1)$ & 0.81 & 331.6 & 321.7 \\
\hline \hline
\end{tabular}

quadrupole) are expected to be small in the same region. Moreover, three-body terms in the van der Waals trimer are usually slightly attractive at short distances, therefore weakening the repulsive interaction, and only slightly repulsive at distances larger than the equilibrium one. Besides, in a previous work ${ }^{19}$ we showed that also in the case of $\mathrm{He}_{2} \mathrm{H}^{-}$, where a charge-induced dipole-induced dipole effect is present, this accounts only for less than $1 \%$ of the total interaction energy.

As to $\Psi_{T}(\mathbf{R})$, our trial wave function has the common form

$$
\Psi_{T}(\mathbf{R})=\prod_{i<j}^{n} \psi\left(r_{i j}\right) \prod_{i}^{n} \phi\left(r_{i}\right),
$$

where no one-body part was used, and

$$
\begin{aligned}
\psi(r)=\phi(r)= & \exp \left\{-\frac{p_{5}}{r^{5}}-\frac{p_{3}}{r^{3}}-\frac{p_{2}}{r^{2}}-p_{1} r-p_{0} \ln (r)\right. \\
& \left.+a \exp \left[-b\left(r-r_{0}\right)^{2}\right]\right\} .
\end{aligned}
$$

Here, with respect to the usual Rick-Lynch-Doll form ${ }^{20,21}$ we added two more terms in the exponent to improve the overall quality of the trial function. Specifically, whereas $-p_{3} / r^{3}$ was added to reduce the fluctuation of the local energy in the repulsive region of the pair potential as previously done, ${ }^{19}$ the $a \exp \left[-b\left(r-r_{0}\right)^{2}\right]$ term was introduced to improve the second shell description at the VMC level, as suggested by Reatto. ${ }^{22}$

The parameters of the model wave function were fully optimized minimizing the variance of the local energy ${ }^{23,24}$ for each cluster using a fixed set of 5000 configurations. We refer to our previous works ${ }^{19,25}$ for full details of the optimization procedure. The optimized parameters are available from the authors upon request.

The optimized wave functions were successively used to guide a set of 5000 configurations in order to sample the distributions $f_{T}(\mathbf{R})=\Psi_{T}^{2}(\mathbf{R})$ (by means of VMC) or $f_{0}(\mathbf{R})$ $=\Psi_{T}(\mathbf{R}) \Psi_{0}(\mathbf{R})$ (by means of DMC). These distributions were used to compute the mean energy and the exact one using the mixed estimator

$$
\langle\mathcal{H}\rangle_{T(0)}=\frac{\int f_{T(0)}(\mathbf{R}) \mathcal{H}_{\mathrm{loc}}(\mathbf{R}) d \mathbf{R}}{\int f_{T(0)}(\mathbf{R}) d \mathbf{R}}
$$

as well as the mixed and second-order estimate $\langle\mathcal{O}\rangle_{\mathrm{SOE}}$ $=2\langle\mathcal{O}\rangle_{0}-\langle\mathcal{O}\rangle_{T}$ of many other expectation values (e.g., the interparticle distribution functions). ${ }^{13}$ The SOE was used in order to reduce the bias introduced in the mixed estimate of operators that do not commute with the Hamiltonian by the use of a nonexact trial wave function. Since the accuracy of our model trial wave function deteriorates upon increasing the number of atoms, we present only SOE results.

\section{RESULTS}

\section{A. Structure and energetics}

The VMC and DMC energies $E(n)$ for the $\mathrm{AgHe}_{n}$ clusters with $n$ up to 100 are shown in Table I. The ratio $E_{\mathrm{VMC}} / E_{\mathrm{DMC}}$ is larger than 0.9 for the clusters including only a first shell (see the following), and progressively decreases during the building of the second shell, evidencing that even the Reatto's term included in the wave function does not give enough flexibility. In Table I, we also report the differential quantity 


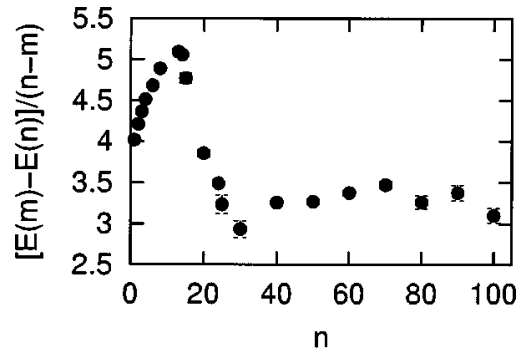

FIG. 2. $\Delta(n)$ values as a function of the number of He atoms.

$$
\Delta(n)=-\left[E_{\mathrm{DMC}}(n)-E_{\mathrm{DMC}}(m)\right] /(n-m) .
$$

$E(m)$ is the energy of the largest cluster $\mathrm{AgHe}_{m}$ having $m$ $<n$. $\Delta(n)$, also shown in Fig. 2, can be interpreted as the evaporation energy of an He atom from the cluster.

Before commenting on the energy results, in order to make the discussion clearer we anticipate that $\mathrm{Ag}$ was found, during our simulations for the largest clusters, to reside close to its geometrical center, therefore indicating its nature of solvated impurity.

The data in Table I and Fig. 2 show that $\Delta(n)$ does not possess a monotonic behavior. Instead, the steady increase for $n<13$ is followed by a rapid decrease in value before plateauing for $n \sim 25$. This behavior could be interpreted invoking different effects. For $n<13$, a newly added He atom experiences the bare Ag interaction potential plus the interaction with the already present $\mathrm{He}$ atoms, which acts positively, increasing the binding energy. Quantitatively, we found the changes of $\Delta(n)$ versus $n$ similar to the ones obtained for $\mathrm{He}_{n},{ }^{21} \mathrm{He}_{n} \mathrm{H}^{-},{ }^{19}$ and $\mathrm{He}_{n} \mathrm{HF} .{ }^{26}$ For the solvated $\mathrm{HF}$ and $\mathrm{Ag}$, this effect seems to be independent of the nature of the doping impurity, so one may interpret it as a dynamical many-body effect of the interacting helium atoms. Here, it is fair to say that another explanation might be valid due to the fluxional nature of the He clusters. This feature allows any He atom in a small cluster to "feel" on average the interaction with all the remaining ones. However, in doped species this may happen only if the impurity resides on the surface (as in the $\mathrm{He}_{n} \mathrm{H}^{-}$case, for instance) so that the $\mathrm{He}$ atoms cluster together. In the following we will show that strongly interacting impurities like $\mathrm{HF}$ and $\mathrm{Ag}$ tightly bind He atoms effectively forbidding the possibility of having one He on top of a second one until the first shell is filled. So, any atom in the Ag first shell can have on average five or six neighbors at most, so that the increase in the chemical potential should stop around $n=6$. Since this is not the case, as shown in Fig. 2, we are left with the possibility that the aforementioned dynamical many-body effect plays a relevant role in defining the energetics of our smaller clusters. However, we feel more computational work should be done in order to better clarify this issue.

Beyond $\mathrm{AgHe}_{13}$, the value of $\Delta(n)$ decreases, indicating the onset of a repulsive interaction. This could be attributed to an "excluded volume" effect, where each new $\mathrm{He}$ is strongly attracted by $\mathrm{Ag}$ in its first coordination shell, but has to "find room" for itself forcing the other atoms to increase their local density, and increasing their average kinetic energy. Finally, for clusters larger than $\mathrm{AgHe}_{25}$, the evapora-
TABLE II. Total energy $\left(\mathrm{cm}^{-1}\right)$ of the $\mathrm{He}_{n}$ clusters, and their $\mathrm{Ag}$ binding energy $E_{\text {bind }}(n)$. Energies for $n=2-8$ are from Ref. 21, for $n=12,13$ are from Ref. 19.

\begin{tabular}{rll}
\hline \hline$n$ & \multicolumn{1}{c}{$E_{\mathrm{He}_{n}}$} & $E_{\text {bind }}(n)$ \\
\hline 2 & $-0.00089(1)$ & $8.232(1)$ \\
3 & $-0.08784(7)$ & $12.510(2)$ \\
4 & $-0.3886(1)$ & $16.723(1)$ \\
6 & $-1.6077(4)$ & $24.870(4)$ \\
8 & $-3.568(2)$ & $32.682(4)$ \\
12 & $-8.746(7)$ & $47.93(1)$ \\
13 & $-10.299(4)$ & $51.55(1)$ \\
20 & $-23.04(1)$ & $70.13(3)$ \\
25 & $-30.6(3)$ & $79.7(3)$ \\
30 & $-42.06(2)$ & $84.05(7)$ \\
40 & $-69.9(2)$ & $88.8(2)$ \\
\hline \hline
\end{tabular}

tion energy remains roughly constant around $3.1-3.5 \mathrm{~cm}^{-1}$, indicating that a new $\mathrm{He}$ atoms experiences a quite different environment than for $n<25$.

The $\Delta(n)$ value for $50 \leqslant n \leqslant 100$ is similar to the one obtained in clusters containing different solvated impurity (see, e.g., Ref. 26 for the case of HF, and Ref. 27 for OCS). However, the behavior of $\Delta(n)$ for small $n$ (i.e., an increase followed by a decrease) more closely resembles the one found in the case of HF as impurity than in the OCS case that almost monotonically decreases to the limiting value. We interpret the finding for $\mathrm{HF}$ as due to the free rotation of this molecule inside the clusters. This means that the He environment feels a rotationally averaged potential so that HF appears like a strongly interacting spherical impurity, hence similar to Ag. Different from HF, the OCS-He interaction potential is characterized by a strong anisotropy with three deep and well separated minima located oppositely to the $\mathrm{O}$ and $\mathrm{S}$ molecular ends, as well as close to the central $\mathrm{C}$ atom. As shown in Ref. 27, due to the anisotropy the He atoms occupy first the miminum close to the $\mathrm{C}$ atom, and only successively the minima close to $\mathrm{O}$ and then to $\mathrm{S}$. Moreover, due to the barrier between the three minima, the He density for the clusters up to 20 helium atoms also shows three wellseparated and sharp peaks in the plane of the $(R, \theta)$ Jacobi coordinates. We believe the different shape and magnitude of the OCS interaction potential to be the cause of the "apparent" absence of the increase in $\Delta(n)$. More specifically, due to the smaller volume available for each $\mathrm{He}$ in the regions of every minimum, it seems possible that the dynamic manybody effect may be compensated by an increase in the average kinetic energy. That this is exactly the case could be shown only by computing this last quantity, or by computing the energy differences ${ }^{27}$ with an increased statistical accuracy, since the effect has a magnitude of roughly $0.1 \mathrm{~cm}^{-1}$.

Another interesting energetical quantity is represented by the binding energy of the Ag atoms to the He clusters $E_{\text {bind }}(n)=E_{\mathrm{He}_{n}}-E_{\mathrm{AgHe}_{n}}$ as a function of $n$. This is shown in Table II for $n$ up to 40, together with the energy of the reference $\mathrm{He}_{n}$ cluster. From these results it emerges that for $\mathrm{AgHe}_{40}$ the $\mathrm{Ag}$ binding energy is not yet fully converged to the limiting $n \rightarrow \infty$ value. An extrapolation to large $n$ gives a binding energy of around $100 \mathrm{~cm}^{-1}$, that is for $n=40$ more than the $80 \%$ of this quantity is already recovered. This re- 


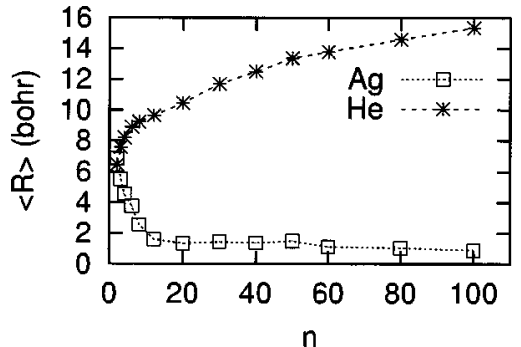

FIG. 3. Average distance of $\mathrm{Ag}$ or $\mathrm{He}$ from the geometrical center of the $\mathrm{He}$ moiety as a function of the number $n$ of He atoms.

sult gives some clue about the maximum number of $\mathrm{He}$ atoms that should leave the cluster in order to dissipate the excess energy released during the binding of $\mathrm{Ag}$ to the droplet. The evaporation energy of a single atom being around $3.3 \mathrm{~cm}^{-1}$, as can be calculated from Table I, this number is around 30 , hence much smaller than the average number of $\mathrm{He}$ atoms in a droplet.

In order to better clarify the structural properties of the Ag doped clusters, during the VMC and DMC simulations we computed average distances and sampled various probability distribution functions for the particle-particle distance and for the distance of an atom from the geometrical center of the cluster

$$
\mathbf{R}_{\mathrm{gc}}^{\mathrm{AgHe}_{n}}=\frac{\sum_{i=1}^{n} \mathbf{r}_{i}+\mathbf{r}_{\mathrm{Ag}}}{n+1},
$$

and the center of the He moiety

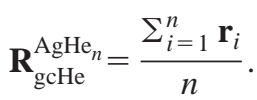

Figure 3 shows the mean value of the $\mathrm{Ag}$ and $\mathrm{He}$ distances from the center of the He moiety (see Eq. (9)) as a function of the number of $\mathrm{He}$ atoms in the cluster. The average distance of He shows a monotonic increase, but the trend is reversed in the $\mathrm{Ag}$ case where a steady decrease of the average distance for $n<20$ is followed by less marked changes. Moreover, a small increase in the average distance of $\mathrm{Ag}$ from the $\mathrm{He}$ center is also visible for the range 30 $\leqslant n \leqslant 50$. The two global trends shown by Fig. 3 clearly support the notion of a solvated $\mathrm{Ag}$ inside the clusters. The steep decrease (increase) of the $\mathrm{Ag}(\mathrm{He})$ distance for $n<20$ is consistent with the formation of a spherical solvatation shell around the impurity, followed by the growing of outer shells. To show that this is just the case, Fig. 4 represents the inter-

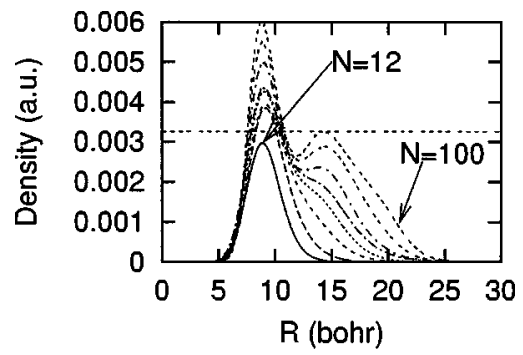

FIG. 4. He density distributions around Ag for $n=12,20,30$, 40, 50, 60, 80, and 100 . The horizontal dashed line represents the pure He bulk density.

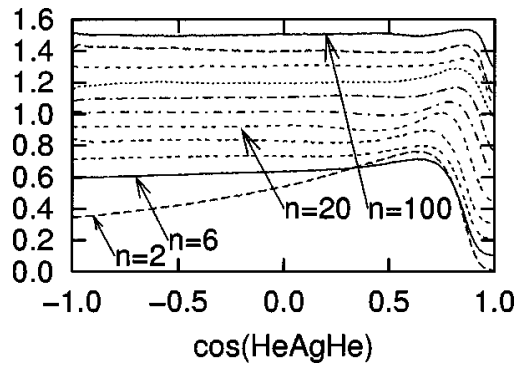

FIG. 5. $\cos (\mathrm{HeAgHe})$ density distributions for $n=2,6,8,12,20,30,40,50$, 60,80 , and 100. Each distribution is shifted upwards with respect to the previous one by 0.1 .

particle $\mathrm{Ag}-\mathrm{He}$ probability density functions for clusters having $n$ from 12 to 100 . These were normalized so that $4 \pi \int_{0}^{\infty} r^{2} \rho(r) d r=n$, therefore representing the local density of He atoms around Ag. The functions for $n<20$ have the same shape as the $\mathrm{AgHe}_{20}$ one, so we plot only $n=12$ in the graph. As to $\mathrm{AgHe}_{30}$, the presence of a broad shoulder at large $r$, which successively develops into a well-defined peak, unambiguously indicates a second shell. More interestingly, the height of the first peak continuously rises until the second shell is completely filled, as indicated by the onset of another shoulder at large $\mathrm{Ag}-\mathrm{He}$ distance for $\mathrm{AgHe}_{100}$. Moreover, the density minimum between the first and second shell peaks also increases in height on going toward larger clusters, becoming just $15 \%$ less than the second shell peak height. This evidence can be interpreted as a direct signature of the "nonrigidity" of the first He shell, as well as of an easy exchange process between the first and second shells. ${ }^{28}$ It is also worth mentioning that, being the Ag solvated in the He moiety, the probability distributions of the He atoms with respect to the geometrical center of the cluster show a marked similarity to the ones presented in Fig. 4, especially for the largest systems.

As to the angular distributions, Fig. 5 shows several $\cos (\mathrm{HeAgHe})$ distributions. The smaller clusters $(n \leqslant 20)$ show a deep minimum for $\cos (\mathrm{HeAgHe})=1$ and a smooth maximum located in the $0.6-0.8$ range, both strong indications of a structured distribution of the He atoms in the first solvation shell. As the overlap of the two atoms is forbidden by their repulsion, the only arrangement having $\cos (\mathrm{HeAgHe})=1$ would be when an $\mathrm{He}$ is on top of a second one, but this possibility is hindered by the strength of the ${ }^{2} \Sigma$ AgHe potential that forces the He motion in a limited radial region around $\mathrm{Ag}$ as shown by the $\mathrm{AgHe}_{12}$ radial distribution. Instead, the smooth maximum indicates the relative localization effects due to the attractive interaction between $\mathrm{He}$ atoms. This effect is particularly evident for $\mathrm{AgHe}_{2}$, whose angular distribution function decreases on going toward $\cos (\mathrm{HeAgHe})=-1$. The position of the maximum shifts to larger $\cos (\mathrm{HeAgHe})$ values on going from $n=2$ to $n=20$, suggesting a progressively more structured packing of the $\mathrm{He}$ atoms in the first shell, and agreeing nicely with the aforementioned "excluded volume" interpretation. The structured packing is also supported by the shallow second peak located around 0.1 in the $\mathrm{AgHe}_{20}$ cosine distribution. Both the mini mum and the maximum are "smeared out" by adding $\mathrm{He}$ 
atoms to $\mathrm{AgHe}_{20}$, a clear indication that the second shell is less structured and more fluid than the first one. However, the persistence in the $\mathrm{AgHe}_{40}$ and $\mathrm{AgHe}_{50}$ angular distributions of the maximum around 0.8 also supports the possibility of having a nonspherical shape of the cluster due to the attractive interaction between the second shell atoms. This may cause the He atoms to stay preferentially closer to each other, therefore preventing the formation of an homogeneous distribution in the second shell, and perhaps also explaining the small increase of the $\mathrm{Ag}$ distance from the center of the He moiety noticed in Fig. 3.

\section{B. Excitation spectra}

As to the absorption spectrum of the embedded $\mathrm{Ag}$ atom, we computed this observable using the semiclassical approach proposed by Cheng and Whaley ${ }^{29}$ for the FranckCondon line shapes of an electronic transition in a condensed phase system. They adapted the semiclassical theory by $\mathrm{Lax}^{30}$ to the quantum Monte Carlo framework by taking into account the temperature of $0 \mathrm{~K}$. In the crudest Lax's approximation, the spectral lines of a chromophore are computed collecting the distribution of the differences $V_{\text {exc }}(\mathbf{R})$ $-V_{\mathrm{gs}}(\mathbf{R})$ over the sampled $f(\mathbf{R})$. This amounts to averaging over all possible thermally populated vibrational states of the system for the electronic ground state. In our case, $V_{\mathrm{gs}}(\mathbf{R})$ $\times\left(V_{\text {exc }}(\mathbf{R})\right)$ is the interaction potential between the ground (excited) state Ag atom with the surrounding $\mathrm{He}$ atoms. At 0 $\mathrm{K}$, the vibrational ground state is the only one populated, and the Franck-Condon line shapes also assume a simple form, equivalent to collecting the distribution of the values $V_{\text {exc }}(\mathbf{R})+\sum_{i<j} V_{\mathrm{HeHe}}\left(r_{i j}\right)-E_{0}$, where $E_{0}$ is the DMC ground state energy (all the details of the derivation of this formula can be found in Ref. 29). In applying this last equation to predict the excitation spectra of Li doped solid $\mathrm{H}_{2}$, Cheng and Whaley were forced to introduce an approximation to $E_{0}$ due to its infinite value in a crystal. In the case of a finite cluster the exact DMC $E_{0}$ result can be "plugged in" the above-mentioned equation. This amounts to running the simulation of a given cluster twice, the first time to obtain the energy and the second time to compute the excitation spectrum. However, it is worth stressing that, different from the energy, the sampled spectrum was found to converge quite rapidly, so that the total computational cost for a given cluster increases only slightly with respect to the energy simulation.

The three $V_{\text {exc }}(\mathbf{R})$ potential energy surfaces (PESs) for a given cluster configuration are obtained from the $\mathrm{AgHe}^{2} \Pi_{1 / 2},{ }^{2} \Pi_{3 / 2}$, and ${ }^{2} \Sigma$ interaction potentials ${ }^{12}$ using the diatomic-in-molecules approach. ${ }^{31,32}$ All the details needed to compute the $\mathrm{Ag}$ excitation spectrum are well described by Nakayama and Yamashita for the isoelectronic valence metals $\mathrm{Li}, \mathrm{Na}$, and $\mathrm{K} .{ }^{15}$ However, for the discussion that will follow, we report two out of the six independent matrix elements needed to compute the total spin-orbit averaged adiabatic potentials. These read

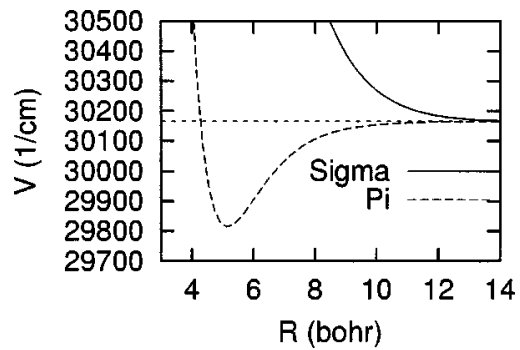

FIG. 6. AgHe $\Sigma$ and $\Pi$ excited states interaction potentials without spinorbit coupling.

$$
\begin{aligned}
\mathbf{U}_{11}=\mathbf{U}_{33}= & \sum_{i=1}^{n}\left[\frac{1+\cos ^{2}\left(\theta_{i}\right)}{2} V_{\Pi}\left(\mathbf{r}_{i \mathrm{Ag}}\right)\right. \\
& \left.+\frac{\sin ^{2}\left(\theta_{i}\right)}{2} V_{\Sigma}\left(\mathbf{r}_{i \mathrm{Ag}}\right)\right]
\end{aligned}
$$

and

$$
\mathbf{U}_{22}=\sum_{i=1}^{n}\left[\sin ^{2}\left(\theta_{i}\right) V_{\Pi}\left(\mathbf{r}_{i \mathrm{Ag}}\right)+\cos ^{2}\left(\theta_{i}\right) V_{\Sigma}\left(\mathbf{r}_{i \mathrm{Ag}}\right)\right],
$$

where $V_{\Pi}\left(\mathbf{r}_{i \mathrm{Ag}}\right)$ and $V_{\Sigma}\left(\mathbf{r}_{i \mathrm{Ag}}\right)$ are the interaction potentials without the spin-orbit coupling, and $\theta_{i}$ is the angle between the $\mathbf{r}_{i}-\mathbf{r}_{\mathrm{Ag}}$ distance vector and the $z$ axis of the laboratory coordinate frame. The $V_{\Pi}\left(\mathbf{r}_{i \mathrm{Ag}}\right)$ and $V_{\Sigma}\left(\mathbf{r}_{i \mathrm{Ag}}\right)$ potentials are shown in Fig. 6. The spin-orbit coupling, responsible for the splitting of the Ag spectrum in the $D_{1}$ and $D_{2}$ lines, is successively introduced, neglecting its distance dependency as indicated in Ref. 12. This approximation is expected to be fairly accurate due to the large average $\mathrm{Ag}-\mathrm{He}$ distances sampled during the simulations.

At this point, we feel a few remarks on the expected accuracy of the approach employed to compute excited state energies are needed. As already pointed out by several authors, ${ }^{33,34}$ this strategy is equivalent to a first-order perturbation theory treatment over the limited basis set composed by three $P$ states. This choice has two major shortcomings: first, it does not allow one to completely include nonadditive three-body effects in the excited PES due to the lack of second-order correction coming from the inclusion of $\mathrm{He}$ excited states in the basis set. Second, it does not allow the possibility of mixing Ag excited states different from the $P$ ones, therefore hindering the possibility of accurately describing the anisotropic distortion of the electronic density. Both these drawbacks might be cured by an higher order perturbation theory approach over an extended basis set containing, at least, the lowest lying $D$ states of Ag. The net effects would be of introducing a higher degree of anisotropy in the excited PES, and of blueshifting the average absorption peak position. However, for the $\mathrm{NaAr}_{n}$ clusters Langhoff ${ }^{34}$ showed that the changes in the computed spectra are not relevant (i.e., few tens of $\mathrm{cm}^{-1}$ ), and we believe this would be the case also for our $\mathrm{AgHe}_{n}$ clusters. This is primarily due to the smaller polarizability of $\mathrm{He}$ than $\mathrm{Ar}$ and to the larger $P-D$ energy difference of $\mathrm{Ag}$ than Na. Also, for our larger clusters the Ag atom is embedded in an highly 


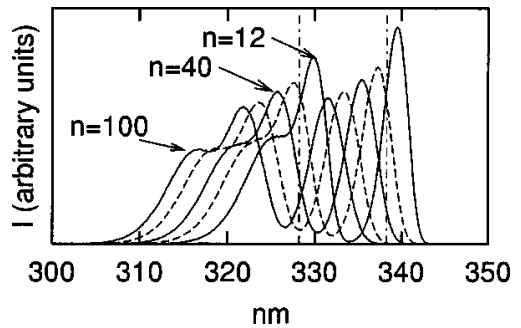

FIG. 7. Simulated absorption spectra for $\mathrm{AgHe}_{n}$ clusters with $n=12,20,40$, 70, and 100. The vertical lines represent the free Ag spectrum.

isotropic environment, so that the mixing of the $D$ states should not play a major role in describing the overall shape of the Ag electron density.

The spectra obtained collecting $V_{\text {exc }}(\mathbf{R})-V_{\text {gs }}(\mathbf{R})$ during the simulations are shown in Table I, and in Fig. 7 for several representative clusters. The same quantities obtained by collecting $V_{\text {exc }}(\mathbf{R})+\Sigma_{i<j} V_{\mathrm{HeHe}}\left(r_{i j}\right)-E_{0}$ are blueshifted by less than $1 \mathrm{~nm}$. The computed spectra clearly show the two separated bands deriving from the excitation of $\mathrm{Ag}$ into ${ }^{2} P_{1 / 2}$ and ${ }^{2} P_{3 / 2}$ states, the second one also displaying the classical short wavelength shoulder typical of the $D_{2}$ line of heavy alkali atoms in superfluid helium. ${ }^{6,7}$ For our largest cluster, the $D_{1}$ and $D_{2}$ lines have maxima located at 331.6 and $321.7 \mathrm{~nm}$, and a full width at half maximum (FWHM) of 4.3 and 9.8 $\mathrm{nm}$, respectively. These results are in accurate agreement with the experimental wavelengths 332.8 and $322.5 \mathrm{~nm}$, and the FWHM 4.0 and $8.5 \mathrm{~nm}^{10}$ we measured from their spectra. It is worth noticing that both the $D_{1}$ and $D_{2}$ lines of the Ag spectrum are strongly blueshifted with respect to the free $\mathrm{Ag}$ ones. This feature indicates that for both the ${ }^{2} P_{1 / 2}$ and ${ }^{2} P_{3 / 2} \mathrm{Ag}$ states the overall interaction with the surrounding $\mathrm{He}$ atoms is repulsive when embedded in the He distribution generated by the Ag ground state. The destabilization of the $\mathrm{Ag}^{2} P_{1 / 2}$ and ${ }^{2} P_{3 / 2}$ states with respect to the free $\mathrm{Ag}$ can be quantified using our binding energy results for the ground state together with the computed or the experimentally recorded spectra. Accepting as limiting value $E_{\text {bind }}$ $=100 \mathrm{~cm}^{-1}$ for $n \rightarrow \infty$, one can use the $D_{1}$ and $D_{2}$ absorption lines in Table I to compute the binding energy of the excited Ag to the He clusters using

$$
E_{\mathrm{bind}}^{\mathrm{exc}}=E_{\mathrm{bind}}-\left(h \nu_{\mathrm{solv}}-h \nu_{\mathrm{vac}}\right)
$$

so that one obtains $E_{\text {bind }}{ }^{2} P_{1 / 2} \sim-497 \mathrm{~cm}^{-1}$ and $E_{\text {bind }}{ }^{2} P_{3 / 2}$ $\sim-506 \mathrm{~cm}^{-1}$. These two values indicate that the break up of the excited system into the excited $\mathrm{Ag}$ atom and the helium moiety is energetically the most stable dissociation channel. Also, this finding supports the possibility that after the excitation of the embedded $\mathrm{Ag}$, this could leave the cluster, ${ }^{11}$ converting the excess energy into kinetic energy if just the excited state would survive long enough. Moreover, these results give quantitative support to the mechanism proposed in Ref. 11 to explain the recording of the Rydberg series of the ${ }^{2} P_{1 / 2} \mathrm{Ag}$ state after the first excitation of $\mathrm{Ag}$ inside the clusters. In that case, the excited Ag seemed to be pushed out after the photon absorption, and this mechanism has been indicated ${ }^{11}$ as a possible way to inhibit the growth of metal clusters inside the He droplet and, therefore, to se- lect its number of metal atoms. However, it is also possible for excited Ag to convert the excess energy exciting the vibrational modes of the He atoms of the cluster. This mechanism would probably produce a partial break up of the He moiety that could lose up to $\sim 500 / 3.3 \sim 151$ atoms. In this case, the dynamics of the excited Ag would be determined only by the shape of the global interaction potential after the dissipation of the excess energy by the cluster. In particular for the ${ }^{2} P_{1 / 2}$ state, it is highly probable that this would interact repulsively with all the He atoms in the first shell due to the effect of the spin-orbit coupling as indicated in Ref. 15 for the $\mathrm{Na}$ and $\mathrm{K}$ atoms. So, the net effect would be, again, to push Ag outside the cluster. This idea is also consistent with the fact that no exciplexes correlated to the ${ }^{2} P_{1 / 2}$ were found in liquid $\mathrm{He}^{5}$ indicating that after the excitation and dissipation there is no pathway that could lead to the exciplex formation. These arguments cannot be used in the case of the ${ }^{2} P_{3 / 2}$ state, so that we believe a quantum dynamical simulation of the process to be mandatory in order to shed some light on this problem.

From the spectra shown in Fig. 7, it clearly appears that the broadening of the absorption bands increases on going toward larger clusters. This evidence indicates that the Ag electronic degrees of freedom are coupled with the motion of an increasing number of $\mathrm{He}$ atoms, and not only with those located in the first shell. More interestingly, whereas all the clusters with $n \leqslant 15$ show a redshift with respect to the free Ag lines, the ones with $n \geqslant 19$ display a blueshift strongly dependent on the number of He atoms. Here, the redshift for $n \leqslant 15$ indicates that the clusters possess an internal distribution such that a vertical transition brings them in a region of the excited state potential where the complexes can form a bound state. This may give the possibility of producing $\mathrm{AgHe}_{n}(n=1-15)$ exciplexes starting from the corresponding clusters, and of studying experimentally their spectrum and decaying dynamics. Conversely, the larger clusters are vertically excited to repulsive regions of the PES, therefore preventing the direct formation of larger exciplexes.

The blueshift for $n \geqslant 19$, at variance with basic solvation concepts, indicates a large effect of the second shell filling on the absorption wavelengths. This is confirmed by the computational evidence that the excitation spectrum of $\mathrm{AgHe}_{100}$, which shows the onset of a third shell, closely agrees with the one of $\mathrm{AgHe}_{90}$ (see Table I). In order to rationalize this observation, as well as the monotonic blueshift of the absorption bands upon increasing of $n$, one must notice that the portion of the AgHe pair distribution located in the 10-13 bohr range overlaps with the tail of the repulsive excited $\mathrm{AgHe}^{2} \Sigma$ potential (see Fig. 6). As a consequence, this zone of the pair density introduces a net positive contribution to the diagonal elements of the matrix [see Eqs. (10) and Eq. (11)] whose eigenvalues define the three electronic excited PESs of the complexes. Since the magnitude of these contributions is dependent on the local He density via the sum $\sum V_{\Sigma}(r)$ over the He atoms falling in that range, there is a net increase of the values of the diagonal elements upon increasing the size of the cluster. This fact reflects itself in a positive shift of the eigenvalues, and hence in the blueshift of 
the computed spectra. Besides, the computed spectra (see Fig. 7) also show the interesting feature that the high energy tail of the $D_{1}$ excitation band overlaps more with the low energy part of the $D_{2}$ one the larger the cluster. This feature was also present in the spectra recorded in Ref. 10, but its presence was not pointed out by the authors. In principle, this energetic overlap gives the ${ }^{2} P$ states of the embedded $\mathrm{Ag}$ a chance to couple together, therefore indicating that the vibrational assisted depopulation of $D_{2}$ is energetically allowed. Previously, Dupont-Roc ${ }^{35}$ proposed a mechanism that explained the absence of the fluorescent emission of light alkali atoms in He by invoking the quenching of the lowest $P$ state due to a strong binding interaction with the surrounding He atoms, but that suggestion failed to account for the absence of the fluorescent $D_{2}$ line in the heavier alkali metals. At variance with this, the energetic overlap between the two broad absorption bands found in our spectra may explain both the presence of the $D_{1}$ and the absence of $D_{2}$ bands in the fluorescence spectrum while exciting the $D_{2}$ line, ${ }^{5}$ and the fact that only the ${ }^{2} P_{1 / 2}$ Rydberg series has been recorded in Ref. 11. Again, a quantum dynamical simulation appears to be mandatory in order to more thoroughly define the mechanism.

\section{CONCLUSIONS}

In this study, we investigated the energetics and structure of $\mathrm{Ag}$ doped He clusters by means of the VMC and DMC techniques. These methods are well suited for these clusters since the thermal effects are expected to play only a minor role in defining their properties. Moreover, we also simulated the $\operatorname{Ag}^{2} P_{1 / 2} \leftarrow{ }^{2} S_{1 / 2}$ and ${ }^{2} P_{3 / 2} \leftarrow{ }^{2} S_{1 / 2}$ excitation spectra for various clusters, highlighting the unusual behavior of the frequency shifts with respect to the free atom excitations versus the number $n$ of He atoms, namely negative for $n<15$ and positive for $n>19$. For our largest clusters, the locations of the two maxima of the computed spectrum were found in accurate agreement with the experimental ones, differing by less than a $1 \mathrm{~nm}$. This small residual discrepancy, which can be accounted for by some inaccuracy in the interaction potentials between the excited state of $\mathrm{Ag}$ and $\mathrm{He}$, in our view indicates the effectiveness of the theoretical approach employed to model the excitation process. This finding allows one to tackle the modeling of more complicated doping impurities, like metal dimers and small clusters, as well as small organic molecules, with good confidence.

Based on our results, we also propose that small $\mathrm{AgHe}_{n}$ clusters, excited by means of light source, could lead to the formation of exciplexes containing up to $15 \mathrm{He}$ atoms, and that the excited Ag could be "spited out" by larger clusters. Moreover, the partial energetic overlap between the simulated $D_{1}$ and $D_{2}$ excitation bands seems to indicate that a vibrational relaxation between the two ${ }^{2} P$ states is possible. However, a detailed simulation of the internal cluster dynamics is mandatory in order to clarify all the details of the processes involved after the metal excitation.

\section{ACKNOWLEDGMENTS}

The authors thank Professor Michio Takami for sending the computed interaction potentials. This work was supported by the Italian MIUR Grant No. MM03265212. The authors are indebted to the Istituto CNR di Scienze e Tecnologie Molecolari (ISTM) for grants of computer time.

${ }^{1}$ J. P. Toennies and A. F. Vilesov, Annu. Rev. Phys. Chem. 49, 1 (1998).

${ }^{2}$ K. Nauta and R. E. Miller, Science 287, 293 (2000); 283, 1895 (1999).

${ }^{3}$ Y. Kwon, P. Huang, M. V. Patel, D. Blume, and K. B. Whaley, J. Chem. Phys. 113, 6469 (2000).

${ }^{4}$ Y. Takahashi, K. Sano, T. Kinoshita, and T. Yabuzaki, Phys. Rev. Lett. 71, 1035 (1993).

${ }^{5}$ J. L. Persson, Q. Hui, Z. J. Jakubek, M. Nakamura, and M. Takami, Phys. Rev. Lett. 76, 1501 (1996).

${ }^{6}$ T. Kinoshita, K. Fukuda, Y. Takahashi, and T. Yabuzaki, Phys. Rev. A 52, 2707 (1995).

${ }^{7}$ T. Kinoshita, K. Fukuda, and T. Yabuzaki, Phys. Rev. B 54, 6600 (1996).

${ }^{8}$ S. Ogata, J. Phys. Soc. Jpn. 68, 2153 (1999).

${ }^{9}$ Z. J. Jakubek, Q. Hui, and M. Takami, Phys. Rev. Lett. 79, 629 (1997).

${ }^{10}$ A. Bartelt, J. D. Close, F. Federmann, N. Quaas, and J. P. Toennies, Phys. Rev. Lett. 77, 3525 (1996).

${ }^{11}$ F. Federmann, K. Hoffmann, N. Quaas, and J. D. Close, Phys. Rev. Lett. 83, 2548 (1999).

${ }^{12}$ Z. J. Jakubek and M. Takami, Chem. Phys. Lett. 265, 653 (1997).

${ }^{13}$ B. L. Hammond, W. A. Lester, Jr., and P. J. Reynolds, Monte Carlo Methods in Ab Initio Quantum Chemistry, 1st ed. (World Scientific, Singapore, 1994).

${ }^{14}$ D. Blume and C. H. Greene, J. Chem. Phys. 112, 8053 (2000).

${ }^{15}$ A. Nakayama and K. Yamashita, J. Chem. Phys. 114, 780 (2001).

${ }^{16}$ K. T. Tang, J. P. Toennies, and C. L. Yiu, Phys. Rev. Lett. 74, 1546 (1995).

${ }^{17}$ F. L. Tobin and J. Hinze, J. Chem. Phys. 63, 1034 (1975).

${ }^{18}$ Z. Yan, J. F. Babb, A. Dalgarno, and G. W. F. Drake, Phys. Rev. A 54, 2824 (1996)

${ }^{19}$ M. Casalegno, M. Mella, G. Morosi, and D. Bressanini, J. Chem. Phys. 112, 69 (2000).

${ }^{20}$ S. W. Rick, D. L. Lynch, and J. D. Doll, J. Chem. Phys. 95, 3506 (1991).

${ }^{21}$ M. Lewerenz, J. Chem. Phys. 106, 4596 (1997).

${ }^{22}$ L. Reatto, Nucl. Phys. A 328, 253 (1979).

${ }^{23}$ A. A. Frost, J. Chem. Phys. 41, 478 (1964).

${ }^{24}$ H. Conroy, J. Chem. Phys. 41, 1327 (1964).

${ }^{25}$ D. Bressanini, M. Zavaglia, M. Mella, and G. Morosi, J. Chem. Phys. 112, 717 (2000).

${ }^{26}$ D. Blume, M. Lewerenz, F. Huisken, and M. Kaloudis, J. Chem. Phys. 105, 8666 (1996).

${ }^{27}$ F. Paesani, F. A. Gianturco, and K. B. Whaley, J. Chem. Phys. 115, 10225 (2001).

${ }^{28}$ M. Buzzacchi, D. E. Galli, and L. Reatto, Phys. Rev. B 64, 094512 (2001).

${ }^{29}$ E. Cheng and K. B. Whaley, J. Chem. Phys. 104, 3155 (1996).

${ }^{30}$ M. Lax, J. Chem. Phys. 20, 1752 (1952).

${ }^{31}$ F. O. Ellison, J. Am. Chem. Soc. 85, 3540 (1963).

${ }^{32}$ J. C. Tully and C. M. Truesdale, J. Chem. Phys. 65, 1002 (1976).

${ }^{33}$ J. A. Boatz and M. E. Fajardo, J. Chem. Phys. 101, 3472 (1994).

${ }^{34}$ P. W. Langhoff, J. Phys. Chem. 100, 2974 (1996).

35 J. Dupont-Roc, Z. Phys. B: Condens. Matter 98, 383 (1995). 